https://helda.helsinki.fi

\title{
Anomalous isotopic effect on vibrational properties of $\mathrm{HXeOH}$
}

\section{Khriachtchev, Leonid}

American Institute of Physics

2002-03-22

Journal of Chemical Physics March 22, 2002 Volume 116, Issue 12, pp. 4758-4761

http://link.aip.org/link/?JCP/116/4758/1

Downloaded from Helda, University of Helsinki institutional repository.

This is an electronic reprint of the original article.

This reprint may differ from the original in pagination and typographic detail.

Please cite the original version. 


\title{
Anomalous isotopic effect on vibrational properties of $\mathrm{HXeOH}$
}

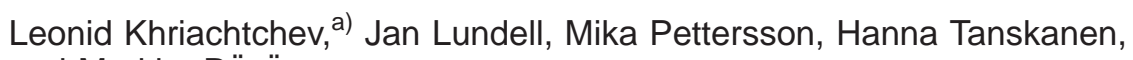 \\ and Markku Räsänen \\ Laboratory of Physical Chemistry, P.O. Box 55, University of Helsinki, FIN-00014 Helsinki, Finland
}

(Received 8 January 2002; accepted 22 January 2002)

\begin{abstract}
We study IR absorption spectra of various isotopomers of the $\mathrm{HXeOH}$ molecules isolated in solid $\mathrm{Xe}$ at $7 \mathrm{~K}$. Upon the ${ }^{16} \mathrm{O}$ to ${ }^{18} \mathrm{O}$ isotopic substitution, we found experimentally an anomalous shift of the $\mathrm{H}-\mathrm{Xe}$ stretching vibration frequency of $\mathrm{HXeOD}$ and $\mathrm{HXeOH}$. This spectral behavior, when the fundamental frequency shifts up in energy for the heavier oxygen isotope, cannot be understood on the basis of the harmonic approximation for fundamental vibrations, and the anharmonic coupling between normal modes is presumably responsible for this anomalous effect. Our anharmonic CC-VSCF calculations describe correctly the experimental observations. (C) 2002 American Institute of Physics. [DOI: 10.1063/1.1459703]
\end{abstract}

Vibrational spectroscopy is a sensitive probe of potential energy surfaces (PES) that determine dynamics of nuclear motion in molecules. The analysis of vibrational spectra allows prediction of structural and thermodynamical properties of matter. In addition to experimental data, theoretical analysis based on electronic structure theory is valuable here. For small molecules, one can obtain an accurate analytical fit of a multidimensional PES by using extensive ab initio electronic structure calculations and apply it then in theoretical vibrational spectroscopy. ${ }^{1}$ For more complicated systems, the harmonic vibrational approximation is usually employed, but this does not provide often deep enough insight. In order to extend the applicability of theoretical analysis, an anharmonic method has recently been developed, which combines electronic $a b$ initio calculations with the vibrational selfconsistent field (VSCF) method and its extension employing perturbation theory (CC-VSCF). ${ }^{2,3}$ In this approach, interactions between pairs of normal modes are taken into consideration, and interactions of triples or higher are neglected.

A large number of novel charge-transfer $\mathrm{HRgY}$ molecules $(\mathrm{H}=$ hydrogen; $\mathrm{Rg}=\mathrm{Ar}, \mathrm{Kr}, \mathrm{Xe}$; $\mathrm{Y}=$ an electronegative fragment) have been characterized by using experimental vibrational spectroscopy in rare-gas solids and ab initio theoretical methods. ${ }^{4,5}$ From the energetic point of view, these molecules are quite unusual due to the low dissociation energy $(\sim 0.4-1.5 \mathrm{eV})$, and we were able to photodecompose one of them (HXeI) directly by exciting the second $\mathrm{H}-\mathrm{Xe}$ stretching overtone at $\sim 3000 \mathrm{~cm}^{-1}$ with intense narrowband IR radiation. ${ }^{6}$ The shallowness of the potential well suggests the importance of anharmonic effects in their ground-state dynamics. It follows that one should carefully account the anharmonicity of various normal modes and the anharmonic couplings between the modes when treating these molecules theoretically. We have recently applied the CC-VSCF approach to HXeI, and a number of exceptionally strong combination and overtone bands predicted theoreti-

a)Electronic mail: Leonid.Khriachtchev@Helsinki.Fi cally have been found in the experimental spectra. ${ }^{7}$ The $\mathrm{HRgY}$ molecules are interesting in many other respects as well. For instance, they constitute an important intermediate during photodissociation of HY molecules in rare-gas hosts influencing the local photodissociation dynamics, ${ }^{8}$ and this process has recently been modeled theoretically for photodissociation of $\mathrm{HCl}$ in Xe clusters. ${ }^{9}$

The anharmonic character of the HRgY molecules suggests search for exceptional effects in their ground-state dynamics. On one hand, we should precisely look at changes of the vibrational properties upon isotopic substitution because the anharmonic mode coupling might be very essential here. On the other hand, the isotopic substitution can test adequateness of the theory employed to obtain the multidimensional PES. The "trivial" harmonic isotopic effect usually considered in spectroscopic studies is a downshift of the fundamental vibrational frequencies when the reduced mass increases. How general is this rule? This question is, in addition to purely fundamental interest, of importance for practical vibrational spectroscopy. In particular, the heavyatom substitution has been successfully employed in many studies, from laboratory identification of unusual species, ${ }^{5,10}$ to astrophysics analysis. ${ }^{11}$ One experimental gas-phase work $^{12}$ reports a small anomalous shift $\left(\sim 0.05 \mathrm{~cm}^{-1}\right)$ of the $(\mathrm{ClHCl})^{-}$anion $\nu_{3}$ fundamental frequency upon ${ }^{35} \mathrm{Cl}$ to ${ }^{37} \mathrm{Cl}$ substitution, and their tentative interpretation was based on symmetry changes. In the present study, we found experimentally and interpreted theoretically the anomalous shifts of $\mathrm{H}-\mathrm{Xe}$ stretching vibrational frequencies of $\mathrm{HXeOH}$ and $\mathrm{HXeOD}$ upon oxygen isotopic substitution when the fundamental frequencies increased with an increase of mass. We demonstrate that it is impossible to interpret this effect on the basis of harmonic approximation, and the use of anharmonic theory is crucial for qualitatively correct insight.

The experimental procedure to prepare the HRgY molecules includes UV solid-state photodissociation of a HY precursor followed by thermal mobilization of hydrogen atoms in the rare-gas lattice. ${ }^{4}$ In order to prepare $\mathrm{H}_{2} \mathrm{O} / \mathrm{Xe}$ solid mixtures in the present experiments, we co-deposited water 


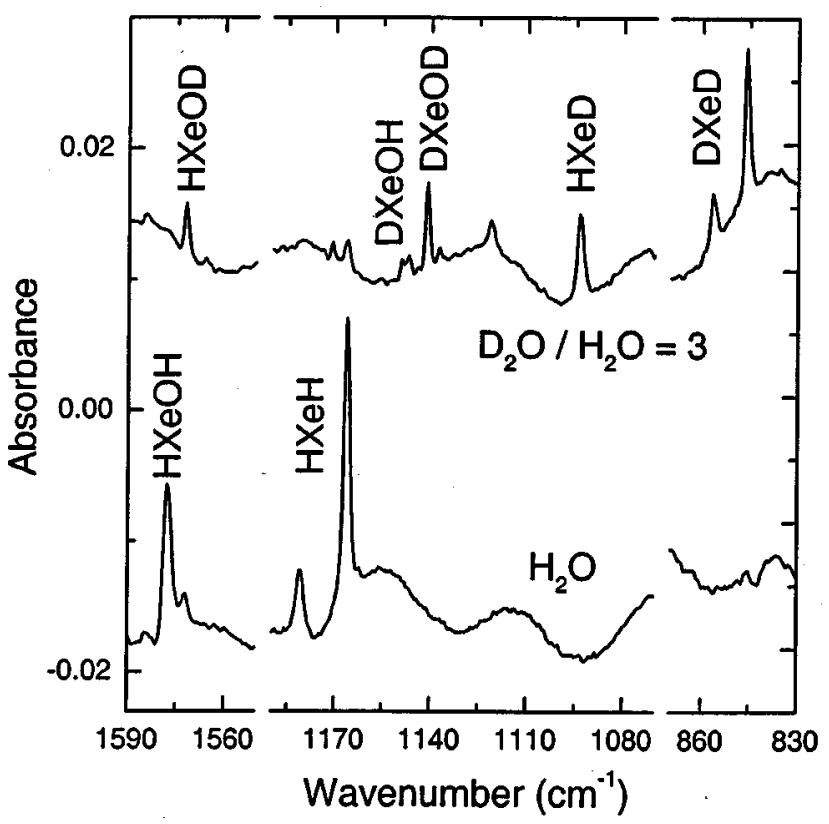

FIG. 1. IR absorption spectra of Xe-containing molecules prepared from water and Xe. The $\mathrm{H}_{2} \mathrm{O} / \mathrm{Xe}$ solid mixtures were first irradiated at $193 \mathrm{~nm}$ and then annealed at $45 \mathrm{~K}$. The spectra were measured at $7 \mathrm{~K}$. The smooth background line is introduced by interference of light in the thin matrix layer.

vapor $\left(\mathrm{H}_{2}{ }^{16} \mathrm{O}, \mathrm{D}_{2}{ }^{16} \mathrm{O}, \mathrm{H}_{2}{ }^{18} \mathrm{O}\right.$, and $\left.\mathrm{D}_{2}{ }^{18} \mathrm{O}\right)$ and xenon gas from two separate nozzles onto a CsI window. The deposition temperature was $30 \mathrm{~K}$, the deposition time was $\sim 1 \mathrm{~h}$, and the typical sample thickness was $100 \mu \mathrm{m}$. The measurements were performed at temperatures down to $7 \mathrm{~K}$ in a closed-cycle helium cryostat (DE-202A, APD). The IR absorption spectra in the 4000 to $400 \mathrm{~cm}^{-1}$ region were recorded with a Nicolet 60 SX FTIR spectrometer in most cases by using resolution of $1 \mathrm{~cm}^{-1}$ and co-adding 500 scans. The as-deposited $\mathrm{H}_{2} \mathrm{O} / \mathrm{Xe}$ samples are quite monomeric, ${ }^{13}$ and no complexes with normal matrix impurities like $\mathrm{N}_{2}$ are visible. In the experiments with isotopically substituted water, ordinary water $\left(\mathrm{H}_{2}{ }^{16} \mathrm{O}\right)$ always interferes in the spectra with a typical ratio of $\sim 1 / 3$. In order to decompose water molecules to $\mathrm{H}+\mathrm{OH}$, the $\mathrm{H}_{2} \mathrm{O} / \mathrm{Xe}$ solid mixtures were irradiated with an excimer laser (MPB, MSX-250) operating at $193 \mathrm{~nm}$. Typically, we used in photolysis $\sim 2$ $\times 10^{3}$ pulses with a pulse energy density of $\sim 10 \mathrm{~mJ} / \mathrm{cm}^{2}$. Under irradiation at $193 \mathrm{~nm}$, water decomposes and the IR absorption band of isolated $\mathrm{OH}$ radicals appears in the spectra. ${ }^{14}$ Upon annealing of a photolyzed sample at $\sim 40 \mathrm{~K}$, hydrogen atoms become globally mobile, and $\mathrm{HXeH}$ (1166 and $\left.1180 \mathrm{~cm}^{-1}\right)$ and $\mathrm{HXeOH}\left(1577 \mathrm{~cm}^{-1}\right)$ are formed (see Fig. 1). For the D-enriched samples, DXeH (1094 and 1121 $\left.\mathrm{cm}^{-1}\right), \mathrm{DXeD}\left(846\right.$ and $\left.857 \mathrm{~cm}^{-1}\right), \mathrm{HXeOD}\left(1572 \mathrm{~cm}^{-1}\right)$, DXeOH $\left(1149 \mathrm{~cm}^{-1}\right)$, and DXeOD $\left(1141 \mathrm{~cm}^{-1}\right)$ are known to rise in annealing. ${ }^{14,15}$ It should be mentioned that these Xe-containing molecules exhibit some matrix site structure as seen in Fig. 1, and our data below were obtained for the strongest site bands.

In the experiments, we carefully analyzed the changes of the $\mathrm{H}-\mathrm{Xe}$ stretching frequency of various $\mathrm{HXeOH}$ isotopomers concentrating on the effect of oxygen isotopic substitution. The IR absorption bands were fitted with Gaussian functions and appropriate backgrounds. Most importantly, the $\mathrm{H}-\mathrm{Xe}$ stretching frequency of $\mathrm{HXeOH}$ and $\mathrm{HXeOD}$ is found to be lower for the lighter oxygen isotope (see Table I and Fig. 2 for HXeOD), which is considered as anomalous behavior. For other isotopomers, oxygen isotopic substitution in $\mathrm{DXeOH}$ produces no measurable shift of the $\mathrm{D}-\mathrm{Xe}$ stretching mode, and the effect on the D-Xe stretching mode of DXeOD is quite large $\left(\sim 3 \mathrm{~cm}^{-1}\right)$ and has the normal direction down in energy for the heavier mass.

The reliability of the experimentally detected anomalous shifts should be discussed because the bandwidths in question $\left(\sim 2 \mathrm{~cm}^{-1}\right)$ are rather larger than the observed anomalous shifts. However, the experimental results are completely repeatable. In order to demonstrate this repeatability, we list the actual fitting realizations for the $\mathrm{H}-\mathrm{Xe}$ stretching band centers of HXeOD. For the ${ }^{16} \mathrm{O}$ isotope, they are 1572.18, $1572.15,1572.13$, and $1572.11 \mathrm{~cm}^{-1}$ whereas for the ${ }^{18} \mathrm{O}$-enriched samples, they are $1572.40,1572.43,1572.41$, and $1572.39 \mathrm{~cm}^{-1}$. The typical error given by the leastsquare fitting procedure is $0.02 \mathrm{~cm}^{-1}$. In order to estimate the possible influence of local matrix morphology on the vibrational frequency, we analyzed the absorption frequency of the oxygen-free HXeD molecule in the same experiments, and the typical results are 1093.77, 1093.73, 1093.72, and $1093.72 \mathrm{~cm}^{-1}$, i.e., similarly very repeatable. Thus, we feel confident in the main conclusion of the present work that the $\mathrm{H}-\mathrm{Xe}$ stretching mode of $\mathrm{HXe}{ }^{18} \mathrm{OD}$ is reliably higher in energy than that of $\mathrm{HXe}{ }^{16} \mathrm{OD}$. In average, this difference is measured to be $+0.27 \mathrm{~cm}^{-1}$. However, the true band shift might be even somewhat larger than the measured one due to

TABLE I. Experimental and theoretical shifts of the $\mathrm{H}(\mathrm{D})$-Xe stretching frequencies (in $\mathrm{cm}^{-1}$ ) of various $\mathrm{H} / \mathrm{D}$ isotopomers of $\mathrm{HXeOH}$ upon ${ }^{16} \mathrm{O}$ to ${ }^{18} \mathrm{O}$ substitution. For comparison, the shift of the $\mathrm{H}-\mathrm{N}$ stretching frequencies of $\mathrm{HNC}$ upon ${ }^{12} \mathrm{C}$ to ${ }^{13} \mathrm{C}$ substitution is analyzed.

\begin{tabular}{|c|c|c|c|c|c|c|c|c|}
\hline \multirow[b]{2}{*}{ Species } & \multicolumn{2}{|c|}{ Experiment $^{\mathrm{a}}$} & \multicolumn{2}{|c|}{ CC-VSCF theory } & \multicolumn{2}{|c|}{$\begin{array}{l}\text { Anharmonic theory } \\
\text { without coupling }\end{array}$} & \multicolumn{2}{|c|}{ Harmonic theory } \\
\hline & Freq. $\left({ }^{16} \mathrm{O}\right)$ & Shift $\left({ }^{18} \mathrm{O}\right)$ & Freq. $\left({ }^{16} \mathrm{O}\right)$ & Shift $\left({ }^{18} \mathrm{O}\right)$ & Freq. $\left({ }^{16} \mathrm{O}\right)$ & Shift $\left({ }^{18} \mathrm{O}\right)$ & Freq. $\left({ }^{16} \mathrm{O}\right)$ & Shift $\left({ }^{18} \mathrm{O}\right)$ \\
\hline $\mathrm{HXeOH}$ & 1577.65 & +0.13 & 1720.78 & +0.14 & 1762.85 & -0.03 & 1834.63 & -0.02 \\
\hline HXeOD & 1572.14 & +0.27 & 1717.61 & +0.30 & 1760.03 & -0.03 & 1832.64 & -0.01 \\
\hline $\mathrm{DXeOH}$ & 1149.14 & -0.03 & 1250.01 & +0.04 & 1271.84 & -0.10 & 1305.80 & -0.05 \\
\hline DXeOD & 1141.23 & -3.03 & 1247.60 & -1.28 & 1266.90 & -0.06 & 1302.32 & -0.03 \\
\hline $\mathrm{HNC}$ & $3643.1^{\mathrm{b}}$ & $-0.6^{\mathrm{b}}$ & 3634.88 & -0.40 & 3690.83 & -1.21 & 3840.82 & -0.64 \\
\hline
\end{tabular}

${ }^{a}$ The experimental error obtained from the fitting inaccuracy and data distribution is smaller than $( \pm 0.04)$.

${ }^{\mathrm{b}} \mathrm{The} \mathrm{H}-{ }^{12} \mathrm{C}$ stretching frequency and the shift upon ${ }^{12} \mathrm{C}$ to ${ }^{13} \mathrm{C}$ substitution from Ref. 17 (experiment in solid neon). 


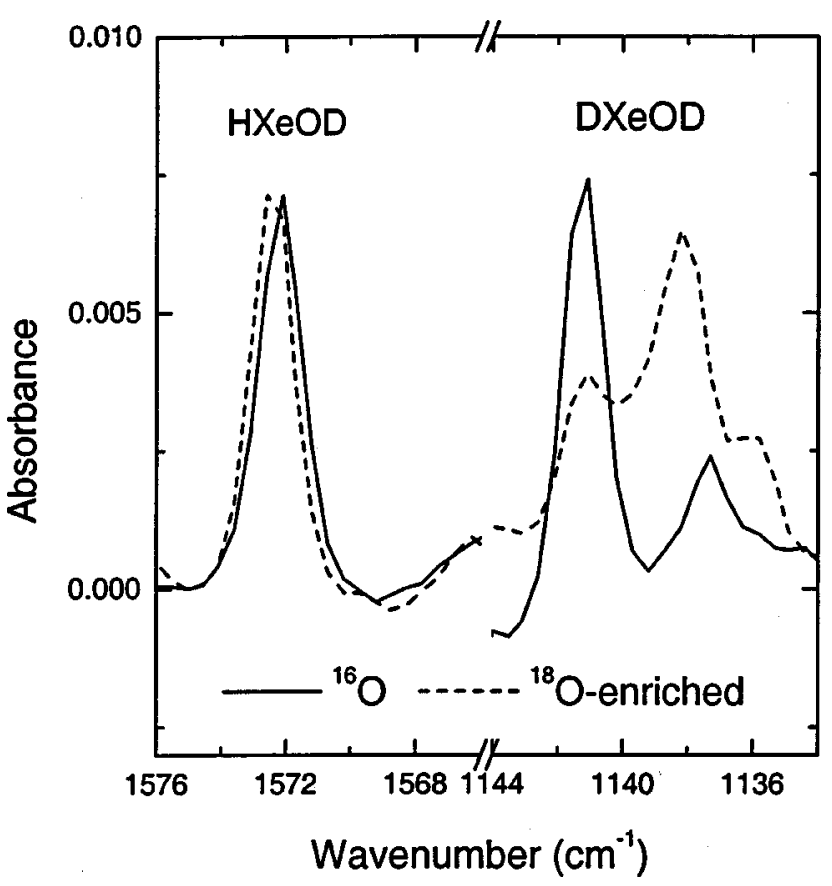

FIG. 2. Effect of oxygen isotopic substitution on fundamental vibrations of Xe-containing molecules. Note the matrix site splitting of the DXeOD band. The contribution of ${ }^{16} \mathrm{O}$ in the ${ }^{18} \mathrm{O}$-enriched sample in seen in the lower frequency interval. The spectra were measured at $7 \mathrm{~K}$.

overlap between the $\mathrm{HXe}^{16} \mathrm{OD}$ and $\mathrm{HXe}{ }^{18} \mathrm{OD}$ bands in the experiments with $\mathrm{D}_{2}{ }^{18} \mathrm{O}$. In fact, we did not mix $\mathrm{D}_{2}{ }^{18} \mathrm{O}$ and $\mathrm{H}_{2}{ }^{18} \mathrm{O}$ on purpose, and $\mathrm{H}$ atoms in the $\mathrm{D}_{2}{ }^{18} \mathrm{O}$ experiments originate simply from residual $\mathrm{H}_{2}{ }^{16} \mathrm{O}$ that is always present in the samples as a natural impurity. It means that the Xecontaining products with the ${ }^{16} \mathrm{O}$ isotope always form in some amounts. The $\mathrm{HXe}{ }^{16} \mathrm{OD} / \mathrm{HXe}{ }^{18} \mathrm{OD}$ proportion is not precisely known, and no reliable deconvolution is possible for such a small frequency difference and a decent signal-tonoise ratio. Furthermore, the bands are broadened limiting the optimal experimental resolution to $1 \mathrm{~cm}^{-1}$. We can mention here that the measured band centers shift down in energy when in some experiments the initial $\mathrm{H}_{2}{ }^{18} \mathrm{O} / \mathrm{H}_{2}{ }^{16} \mathrm{O}$ ratio decreases, and this supports the proposed image. The measured anomalous difference of $+0.13 \mathrm{~cm}^{-1}$ between the $\mathrm{H}-\mathrm{Xe}$ stretching vibrations of $\mathrm{HXe}{ }^{18} \mathrm{OH}$ and $\mathrm{HXe}^{16} \mathrm{OH}$ has a high level of confidence as well.

The structure, energy and vibrational properties of the present Xe-containing molecules have been calculated by the CC-VSCF method. The approach in this work is of the same level of theory as used recently to calculate the anharmonic vibrational spectra of a number of $\mathrm{HRgY}$ molecules. ${ }^{16}$ As emphasized earlier, $\mathrm{HXeOH}$ is quite anharmonic as evidenced, for instance, by the calculated anharmonicity 2 $\times \nu_{2}-2 \nu_{2} \sim 80 \mathrm{~cm}^{-1}$ where $\nu_{2}$ is the $\mathrm{H}-\mathrm{Xe}$ stretching mode and $2 \nu_{2}$ denotes its first overtone. In this situation, the agreement between experimental and calculated fundamental vibrational frequencies can be essentially improved by accounting this property (see Table I) even though the result is not of spectroscopic accuracy yet. We also tested the CCVSCF method for the ${ }^{12} \mathrm{C}$ to ${ }^{13} \mathrm{C}$ isotopic substitutions in HNC molecules comparing the theoretical results with the experimental data available for this molecule. ${ }^{17}$ The idea of this test is to evaluate the CC-VSCF method for the heavy atom substitution in respect with fine perturbation of the $\mathrm{H}-\mathrm{N}$ stretching mode. Importantly, $\mathrm{HNC}$ is also quite anharmonic as judged by the experimental H/D ratio of 1.311 for the $\mathrm{H}-\mathrm{N}$ stretching vibration. Indeed, the harmonic approximation applied to $\mathrm{HNC}$ overestimates strongly the $\mathrm{H}-\mathrm{N}$ stretching vibration energy although the isotopic shift is properly described meaning that the reduced-mass effect still dominates in the isotopic shift of this molecule. The anharmonic approach improves the quantitative agreement with experiment with respect to the fundamental frequencies but it does not feature for HNC qualitatively different results.

Our CC-VSCF results fully support the spectral observations on $\mathrm{HXeOH}$ (see Table I). According to this theory, the $\mathrm{H}-\mathrm{Xe}$ stretching fundamental vibrations of $\mathrm{HXeOH}$ and HXeOD exhibit anomalous up-shifts upon substitution with the heavier oxygen isotope, and the computed shifts agree well with the experiment. In accordance with the experiments, practically no shift for the $\mathrm{H}-\mathrm{Xe}$ stretching mode of $\mathrm{DXeOH}$ and a relatively large downshift for this mode of DXeOD was computationally obtained upon the ${ }^{16} \mathrm{O}$ to ${ }^{18} \mathrm{O}$ substitution. One can see that the harmonic approximation predicts very small but definite downshifts upon substitution with the heavier oxygen isotope in all cases, which qualitatively disagrees with the experiment. This is a case when the harmonic approximation gives qualitatively wrong results. One can speculate, in principle, that the decrease of zero point energy produced by the heavier oxygen isotope makes the molecule more harmonic, which shifts the band up in energy in the case of negligible reduced-mass effect, i.e., it is a purely anharmonic effect. This trend for anharmonicity was found theoretically: the calculated anharmonicities of the $\mathrm{H}-\mathrm{Xe}$ stretching mode are 80.64 and $80.36 \mathrm{~cm}^{-1}$ for $\mathrm{HXe}^{16} \mathrm{OD}$ and $\mathrm{HXe}^{18} \mathrm{OD}$, respectively. However, this change in anharmonicity is insufficient to produce the anomalous shift. Indeed, the anharmonic calculations without accounting for mode couplings are equally wrong (see Table I).

We interpret that the anharmonic mode coupling has a decisive contribution for the anomalous isotopic shifts observed in the present work. The isotopic substitution changes the coupling between normal modes, which changes the vibrational frequencies via the modified coupling coefficients. The contribution of mode coupling to the $\mathrm{Xe}-\mathrm{H}$ stretching fundamental is computationally quite large $\left(\sim 40 \mathrm{~cm}^{-1}\right.$ for $\mathrm{HXeOH}$ ), and its modification upon oxygen isotopic substitution is the reason for the obtained anomalous spectral effect. The analysis for $\mathrm{HXeOH}$ shows that the coupling potentials of the $\mathrm{Xe}-\mathrm{H}$ stretching mode $\left(\nu_{2}\right)$ with the asymmetric bending mode $\left(\nu_{3}\right)$ and the $\mathrm{Xe}-\mathrm{OH}$ stretching mode $\left(\nu_{6}\right)$ change essentially upon the ${ }^{16} \mathrm{O}$ to ${ }^{18} \mathrm{O}$ isotopic substitution. In particular, the coupling of $\nu_{2}$ with $\nu_{3}$ is decreased and the coupling of $\nu_{2}$ with $\nu_{6}$ is increased. It should be also noted that the in-plane bending modes are strongly coupled with the $\mathrm{OH}$ stretching mode and they undergo large changes upon oxygen isotopic substitution as well. Of course, the resulting effect of mode coupling can be also of the "normal" direction as we see for DXeOD, and the mode 
coupling essentially establishes it as well. It should be mentioned that we have explained the anomalous shift in a different way compared with Ref. 12 because their mechanism of triple mode interaction induced by the perturbed $D_{\infty h}$ symmetry is not included into the CC-VSCF model., ${ }^{2,3}$

The relatively large mode coupling in $\mathrm{HXeOH}$ might be connected with the mixed character of its bonding where both ionic and neutral potentials contribute. This Xecontaining molecule dissociates in an unusual way: The detachment of $\mathrm{H}$ destroys the chemical character of the $\mathrm{Xe}-\mathrm{O}$ bond as well, which suggest strong coupling between the $\mathrm{H}-\mathrm{Xe}$ and $\mathrm{Xe}-\mathrm{O}$ stretching coordinates. It should be emphasized that this mode-coupling contribution always competes with the reduced-mass effect, and the anomalous result can occur only when the latter effect is small. It is exactly the case of the Xe containing species due to the large Xe mass, and it can be particularly seen in a very small shift upon oxygen isotopic substitution obtained in the harmonic approximation.

In conclusion, we found experimentally an anomalous shift in the $\mathrm{H}-\mathrm{Xe}$ stretching vibrational frequency of matrixisolated $\mathrm{HXeOD}$ and $\mathrm{HXeOH}$ molecules upon oxygen isotopic substitution when the frequency shifts up in energy for the heavier isotope. This behavior cannot be understood in the framework of harmonic vibrational theory, and the anharmonic coupling between normal modes must be employed to obtain the adequate image. Our anharmonic CC-VSCF calculations describe correctly the experimental observations. This result emphasizes that the effect of anharmonic mode coupling is generally important in vibrational spectroscopy, and it influences vibrational frequencies of various molecules with coupled normal modes.

The Academy of Finland supported this work. R. B. Gerber is thanked for valuable discussions.

${ }^{1}$ S. Skokov, K. A. Peterson, and J. M. Bowman, J. Chem. Phys. 109, 2662 (1998).

${ }^{2}$ G. M. Chaban, J. O. Jung, and R. B. Gerber, J. Chem. Phys. 111, 1823 (1999).

${ }^{3}$ G. M. Chaban, J. O. Jung, and R. B. Gerber, J. Phys. Chem. A 104, 2772 (2000).

${ }^{4}$ J. Lundell, L. Khriachtchev, M. Pettersson, M. Räsänen, Low Temp. Phys. 26, 680 (2000).

${ }^{5}$ L. Khriachtchev, M. Pettersson, N. Runeberg, J. Lundell, M. Räsänen, Nature (London) 406, 874 (2000).

${ }^{6}$ M. Pettersson, J. Nieminen, L. Khriachtchev, and M. Räsänen, J. Chem. Phys. 107, 8423 (1997).

${ }^{7}$ J. Lundell, M. Pettersson, L. Khriachtchev, M. Räsänen, G. M. Chaban, and R. B. Gerber, Chem. Phys. Lett. 322, 389 (2000).

${ }^{8}$ L. Khriachtchev, M. Pettersson, J. Lundell, M. Räsänen, J. Chem. Phys. 114, 7727 (2001).

${ }^{9}$ A. Cohen, M. Y. Niv, and R. B. Gerber, Faraday Discuss. 118, 269 (2001).

${ }^{10}$ B. Liang and L. Andrews, J. Am. Chem. Soc. 123, 9848 (2001).

${ }^{11}$ M. P. Bernstein, S. A. Stanford, and L. J. Allamandola, Astrophys. J. 542, 894 (2000).

${ }^{12}$ K. Kawaguchi, J. Chem. Phys. 88, 4186 (1988).

${ }^{13}$ R. L. Redington and D. E. Milligan, J. Chem. Phys. 39, 1276 (1963).

${ }^{14}$ M. Pettersson, L. Khriachtchev, J. Lundell, and M. Räsänen, J. Am. Chem. Soc. 121, 11904 (1999).

${ }^{15}$ M. Pettersson, J. Lundell, and M. Räsänen, J. Chem. Phys. 103, 205 (1995).

${ }^{16}$ J. Lundell, G. M. Chaban, and R. B. Gerber, J. Phys. Chem. A 104, 7944 (2000).

${ }^{17}$ D. Forney, W. E. Thompson, and M. E. Jacox, J. Chem. Phys. 97, 1664 (1992) 